\title{
Clinical Characteristics of Metastatic Prostate Cancer Patients Infected with COVID-19 in South Italy
}

\author{
Giuseppe Di Lorenzo ${ }^{\mathrm{a}, \mathrm{b}}$ Luciana Buonerba ${ }^{\mathrm{a}}$ Concetta Ingenito $^{\mathrm{a}}$
} Felice Crocetto ${ }^{c}$ Carlo Buonerba $^{d}$ Annamaria Libroia $^{a}$ Antonella Sciarra $^{a}$ Gianluca Ragone $^{\text {a }}$ Roberto Sanseverino $^{\text {e }}$ Simona laccarino ${ }^{a}$ Giorgio Napodano ${ }^{e}$ Ciro Imbimbo $^{c}$ Emilio Leo $^{a}$ Zisis Kozlakidis ${ }^{f}$ Sabino De Placido ${ }^{d}$

a Oncology Unit, "Andrea Tortora" Hospital, ASL Salerno, Pagani, Italy; ${ }^{b}$ Department of Medicine and Health Sciences "Vincenzo Tiberio", University of Molise, Campobasso, Italy; ' Department of Neurosciences, Sciences of Reproduction and Odontostomatology, University of Naples Federico II, Naples, Italy; ${ }^{d}$ Department of Clinical

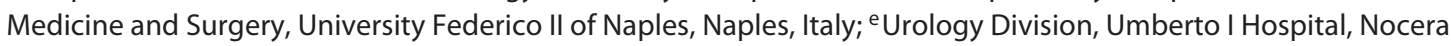

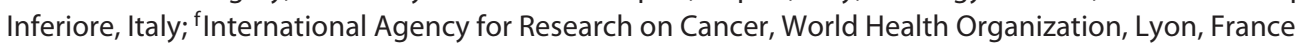

\section{Keywords}

Comorbidities · Coronavirus disease SARS-CoV-2 · Drug

interaction $\cdot$ Hormonal therapy $\cdot$ Prostate cancer

\begin{abstract}
Background: To date, the clinical characteristics of coronavirus disease 19 (COVID-19)-infected urologic cancer patients are unknown. Methods: We have analyzed all patients with prostate cancer undergoing hormonal or chemotherapy treatment and receiving telephone and in person pre-triage between March 1 and 27, 2020, at the Tortora Hospital, Pagani, Italy. Results: Among 72 patients, 48 and 24 were hormone-sensitive (HS) and castration-resistant prostate cancer (CRPC), respectively; $0 \mathrm{HS}$ and $2(8.3 \%)$ CRPC $(p<0.05)$ were positive for COVID-19. Both patients were receiving LHRH agonist therapy, and 1 patient was receiving enzalutamide. Urgent intensive care unit admission was required due to clinical worsening. Blood tests showed severe lym-
\end{abstract}

phopenia, anemia, and an increase in platelets. Retroviral therapy, antibiotics, heparin, and chloroquine were prescribed at the beginning. One patient also received tocilizumab as a salvage treatment. After 3 weeks of hospitalization, the patients were discharged from the hospital. Both patients suffered from an aggressive COVID-19 course due to concomitant comorbidities. Conclusions: Investigating whether hormonal therapy, especially in advanced disease, acts as a protective factor or a risk factor during COVID-19 could be useful.

(c) 2020 S. Karger AG, Basel

\section{Introduction}

Coronavirus infection has become a worldwide problem, with millions of infected people and thousands of deaths. Data from the Italian Health Institute indicate that $26 \%$ of the coronavirus disease 19 (COVID-19) pa-

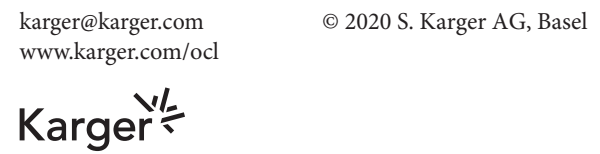


Table 1. Characteristics of 72 prostate cancer patients

\begin{tabular}{ll}
\hline Median age, years & 71 (range 53-82) \\
Comorbidity & $72(100 \%)$ \\
$\quad$ Hypertension & 46 \\
$\quad$ Diabetes & 20 \\
$\quad$ Hepatitis B or C & 5 \\
$\quad$ Osteoporosis & 5 \\
$\quad$ Neurologic disorders & 2 \\
$\quad$ Renal dysfunction & $52(72 \%)$ \\
\hline At least 2 comorbidities & 48 \\
\hline HS disease & 24 \\
\hline CRPC & 72 \\
\hline LHRH & 57 \\
Agonist & 15 \\
Antagonist & 4 \\
\hline Abiraterone & 6 \\
\hline Enzalutamide & 7 \\
\hline Apalutamide & 11 \\
\hline Chemotherapy & 4 \\
Docetaxel & 7 \\
\hline Cabazitaxel &
\end{tabular}

tients had 1 disease, $26 \%$ had 2 diseases, and $47 \%$ had 3 or more diseases, and only $1 \%$ of the patients had no other disease. The most common chronic pre-existing illnesses were arterial hypertension (76\%), ischemic heart disease (37\%), atrial fibrillation (26\%), and active cancer within the previous 5 years (19\%).

As recently reported patients with COVID19 and cancer were more likely to deteriorate into serious illness than those without cancer [1].

The focus of the current manuscript is on patients with an active cancer diagnosis, as these patients require particular clinical attention, starting with the prevention of contact risk for COVID-19 [2].

According to the 2019 data from the Association of Italian Medical Oncology (AIOM), there were 370,000 new cancer diagnoses in Italy, of which 35,000 were prostate cancer diagnoses and $20 \%$ were males $>70$ years. These numbers are in line with the ones reported from previous years [3]. Therefore, considering the high rate of comorbidities, such as hypertension, diabetes, vascular disease in elderly patients [4] as well as the well-known relationship between old age and prostate cancer [5], there is a considerable need to investigate the clinical risk and aggressiveness of COVID-19 in such patients.
The current manuscript describes the clinical characteristics of 72 prostate cancer patients in South Italy, providing a characteristic snapshot of the Italian prostate cancer patient population during the COVID-19 pandemic in Italy.

\section{Methods}

All patients with prostate cancer were included in the present study, being treated with hormonal therapy or chemotherapy; regardless of the stage of disease; of being on a day-hospital visit, hospitalized, or on an outpatient visit. The data was collected between March 1 and 27, 2020, at the Tortora Hospital, Pagani, Italy, coincidental to the height of the first wave of the COVID-19 outbreak in Italy.

The Tortora Hospital is a referral hospital with an average of 1,200 genitourinary cancer patients receiving diagnosis or treatment every year. Among those patients, approximately 500 are prostate cancer patients.

The collection of data involved the use of both telephone and in-person pre-triages. Telephone pre-triage was performed the day before cancer therapy by contacting each patient, as previously described [6]. An in-person pre-triage assessment on the day of cancer therapy provided further checks, including a second interview and temperature measurement.

\section{Results}

Data was collected from all 72 prostate cancer patients attending the Tortora Hospital during the period of the study (March 1-May 27, 2020). The characteristics of the prostate cancer patients who were included in the analysis are shown in Table 1 . Among the 72 patients, 2 patients were confirmed as COVID-19-positive patients (2.8\%) (Table 1) by an reverse transcription-polymerase chain reaction diagnostic test; 0 in the hormone-sensitive (HS) and $2(8.3 \%)$ in castration-resistant prostate cancer (CRPC) setting $(p<0.05)$.

Of those two COVID-19 positive patients; Patient 1 had non-metastatic CRPC with a 3-month PSA double time (DT), thus defining a rapidly evolving disease. He was being treated with an LHRH agonist. Testosterone was suppressed, so the addition of apalutamide was imminent; however, it was not administered as he had to be admitted to the intensive care unit (ICU). This patient was also being followed-up at Federico II University in Napoli (Urology and Oncology Divisions).

Patient 2 had metastatic CRPC and was receiving LHRH therapy plus enzalutamide (Table 2). The patient was treated for 15 months with an LHRH agonist; after asymptomatic bone progression in the lumbar 
Table 2. Clinical characteristics of 2 COVID-19-positive patients

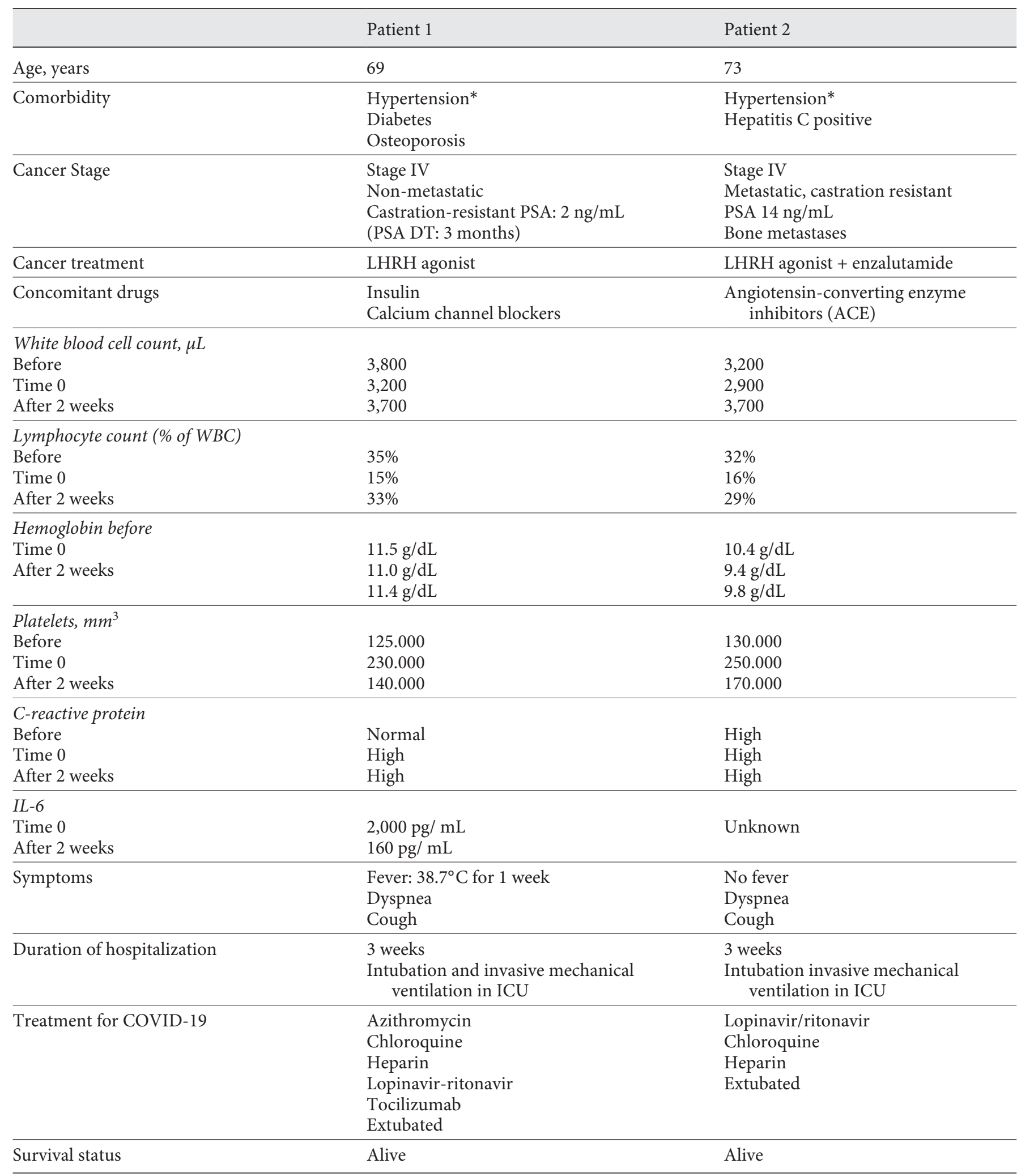

Before: 3 weeks before COVID-19 diagnosis. LHRH, luteinizing hormone-releasing hormone; WBC, white blood CELL count; DT: double time. ${ }^{*}$ Grade 2 hypertension according to NCI common toxicity criteria for 5 years. 
spine and pelvis, enzalutamide was added, ongoing from 5 months.

Urgent hospitalization in the ICU was necessary for both patients due to a clinical worsening picture, in the absence of a COVID-19-positive history by contact or by a prior positive test.

At time 0 , severe lymphopenia, rising levels of platelets, $\mathrm{C}$-reactive protein, fibrinogen and D-Dimer, as well as a peak in the platelet-to-lymphocyte ratio were observed. After admission, IL-6 decreased from $2,000 \mathrm{pg} / \mathrm{mL}$ at baseline to $160 \mathrm{pg} / \mathrm{mL}$. As summarized in Table 2, Patient 1 developed fever, dyspnea, and a cough while Patient 2 developed dyspnea and a cough, but no fever. Both patients were treated with retroviral therapy from the beginning, followed by antibiotics and chloroquine. Chest computed tomography findings revealed ground-glass opacity.

Intubation with invasive mechanical ventilation in the ICU was required for both patients. Patient 1 also received tocilizumab. Three weeks later, both patients were discharged and returned home after three consecutive negative swab tests.

\section{Discussion}

Gillessen and Powles' opinion paper [7] describes how to manage patients with renal, germ-cell, urothelial, and prostate cancer who are COVID-19 positive. The authors concluded that chemotherapy should be delayed and treatment with androgen receptor signaling inhibitors could continue or be prescribed instead of chemotherapy. Based on studies analyzing the frequency of bacterial, viral, and parasitic diseases in patients suffering from hyperglucocorticoidism, testosterone has been shown to have immunosuppressive activity [8].

Therefore, from the literature emerges that testosterone, the most abundant androgen in male organisms, seems to favor the growth of tumors, and in particular prostate cancer due to its negative effect on the immune system which is no longer able to attack the non-self or even generate the pro-inflammatory cytokine cascade [8]. It is possible that suppression of testosterone could lead to upregulation of the immune system, thus resulting in a protective effect against coronavirus infection.

As recently demonstrated by Montopoli et al. [9], prostate cancer patients receiving androgen deprivation therapy had a significantly lower risk of COVID-19 as compared to patients who did not receive androgen deprivation therapy. Our data seem to align with that direction of thought, in the sense that among the HS patients, we have not found any cases, while in a more advanced disease stage, hormone therapy could have a detrimental effect. As suggested by Di Lorenzo, a warning could be issued against androgen receptor signaling inhibitor in the CRPC setting for at least three reasons: concomitant use of prednisone for several months, significant rise in cortisol concentration, and drug interactions [10].

Patient 1 had a rising PSA, a short PSA DT, a low level of testosterone in the blood, and no metastases. Thus, he was considered to be a non-metastatic CRPC patient; therefore, he too received apalutamide. He was being treated with an LHRH agonist. Although apalutamide reduced the levels of testosterone, it could also interfere with retroviral therapy since it is a potent inducer of the CYP450 family [11].

Patient 2 had metastatic CRPC, and so he was treated with an LHRH agonist and enzalutamide. Enzalutamide is an androgen receptor antagonist that suppresses $11 \beta$-hydroxysteroid dehydrogenase- 2 and increases cortisol levels [12]. This leads to an immunosuppressive effect and a negative outcome in the presence of infection. Moreover, cortisol also decreases the number of lymphocytes, which leads to an increased death rate during infection [13]. Enzalutamide is a potent inducer of the cytochrome P450 (CYP) family such as CYP3A4 and a moderate inducer of CYP2C19 and CYP2C9. In vitro evidence shows that enzalutamide also induces CYP2B6 and uridine $5^{\prime}$-diphospho-glucuronosiltransferase 1A1/4 (UGT1A1/4), contributing to antiretroviral metabolism [14]. Hence, if ritonavir and enzalutamide are administered to the same patient, such as in the case of Patient 2, a lower efficacy of ritonavir could be seen.

Patient 2 was not prescribed abiraterone because he was positive for hepatitis $C$ virus, which is characterized by high levels of transaminases, and it is known that a potential side effect of abiraterone is an increase in transaminases. Further, if co-administered with prednisone, abiraterone can have an immunosuppressive effect [15]. Moreover, chemotherapy was not indicated due to the patient's asymptomatic CRPC.

\section{Conclusions}

Whether hormone therapy, especially in a CRPC setting, has a detrimental or beneficial effect during an aggressive course of COVID-19 remains unknown. Reducing the dose of cancer therapy, interrupting treatment if the cancer is not an aggressive cancer, or changing treatment drugs could be options for the management of pros- 
tate cancer patients who are COVID-19 positive [16]. The current manuscript provides the observations from a small sample size of prostate cancer patients. However, these observations seem to align with previously reported results from other parts of the world.

Considering these data an epidemiological study, CO.I.CA. 1 (COVID-19 Infection Cancer patients 1) has been started at several Italian hospitals. The aim of this retrospective/prospective study, which was approved by the relevant ethics committee on April 15, 2020, is to monitor all cancer patients in order to have a better overview of how coronavirus infection influences cancer history.

\section{Acknowledgment}

We would like to thank Editage (www.editage.com) for English language editing.

\section{Statement of Ethics}

Both patients have given their written informed consent to publish their cases. The paper is exempt from ethical committee approval due to the COVID-19 pandemic.

\section{Disclosure Statement}

All authors declare that they have no conflicts of interest to disclose.

\section{Funding Sources}

The authors did not receive any funding.

\section{Author Contributions}

Study concept and design: Giuseppe Di Lorenzo, Luciana Buonerba, Concetta Ingenito.

Acquisition of data: all authors.

Analysis and interpretation of data: all authors.

Drafting of the manuscript: Giuseppe Di Lorenzo, Luciana Buonerba, Concetta Ingenito.

Critical revision of the manuscript for important intellectual content: all authors.

\section{References}

1 Tian J, Yuan X, Xiao J, Zhong Q, Yang C, Liu $\mathrm{B}$, et al. Clinical Characteristics and Risk Factors Associated With COVID-19 Disease Severity in Patients With Cancer in Wuhan, China: A Multicentre, Retrospective, Cohort Study. Lancet Oncol; 2020 May 29;S14702045(20)30309-0. doi: https://doi. org/10.1016/S1470-2045(20)30309-0.

2 Di Lorenzo G, Di Trolio R. Coronavirus Disease (COVID-19) in Italy: Analysis of Risk Factors and Proposed Remedial Measures. Front Med (Lausanne). 2020 Apr;7:140.

3 Association of Italian Medical Oncology (AIOM). Guidelines for Prostate cancer. https://www.aiom.it/wp-content/uploads/2019/10/2019_LG_AIOM_Prostata. pdf. Last access 6 June 2020.

4 Del Pinto R, Ferri C. Hypertension Management at Older Age: An Update. High Blood Press Cardiovasc Prev. 2019 Feb;26(1):27-36.

5 Lu-Yao GL, Kelly WK, Chapman AE. Advanced age and prostate cancer specific mortality among geriatric patients with metastatic prostate cancer. J Clin Oncol. 2018;36(6)suppl: 319 .
6 Ingenito C, Buonerba L, Ferrara C, Busto G, Libroia A, Ragone G, et al. Coronavirus disease 2019 emergency and cancer in the south of Italy. What's new for the oncologist? Front Med (Lausanne). 2020 May; 7:189.

7 Gillessen S, Powles T. Advice Regarding Systemic Therapy in Patients with Urological Cancers During the COVID-19 Pandemic. Eur Urol. 2020 Jun;77(6):667-8.

8 Gubbels Bupp MR, Jorgensen TN. AndrogenInduced Immunosuppression. Front Immunol. 2018 Apr;9:794.

9 Montopoli M, Zumerle S, Vettor R, et al. Androgen deprivation therapies for prostate cancer and risk of infection by SARS-CoV-2: a population-based study $(\mathrm{N}=4532)$. Ann Oncol. 2020 May;S0923-7534(20)39797-0. doi: $\quad$ https://doi.org/10.1016/j.annonc.2020.04.479.

10 Di Lorenzo G. Is it always correct to continue androgen receptor signaling inhibitors in the COVID-19 era? Eur Urol. Forthcoming. 2020.

11 Small EJ, Saad F, Chowdhury S, Oudard S, Hadaschik BA, Graff JN, et al. Apalutamide and overall survival in non-metastatic castration-resistant prostate cancer. Ann Oncol. 2019 Nov;30(11):1813-20.
12 Alyamani M, Li J, Patel M, Taylor S, Nakamura F, Berk M, et al. Deep androgen receptor suppression in prostate cancer exploits sexually dimorphic renal expression for systemic glucocorticoid exposure. Ann Oncol. 2020 Mar;31(3):369-76.

13 Tatsi C, Boden R, Sinaii N, Keil M, Lyssikatos C, Belyavskaya E, et al. Decreased lymphocytes and increased risk for infection are common in endogenous pediatric Cushing syndrome. Pediatr Res. 2018 Feb;83(2):431-7.

14 Nhean S, Bravo J, Sheehan NL, et al. Successful use of the potent enzyme inducer enzalutamide in a treatment-experienced HIV-positive male with prostate cancer. Wolters Kluwer Health; 2018. https://doi.org/10.1097/ QAD.0000000000002019.

15 Colomba E, Marret G, Baciarello G, Lavaud P, Massard C, Loriot $\mathrm{Y}$, et al. Liver tests increase on abiraterone acetate in men with metastatic prostate cancer: natural history, management and outcome. Eur J Cancer. 2020 Apr; 129:117-22.

16 Di Lorenzo G, Di Trolio R, Kozlakidis Z, Busto G, Ingenito C, Buonerba L, et al. COVID 19 therapies and anti-cancer drugs: A systematic review of recent literature. Crit Rev Oncol Hematol. 2020;152:102991. 